\title{
BIAS ISSUES IN CLOSED LOOP IDENTIFICATION WITH APPLICATION TO ADAPTIVE CONTROL*
}

\author{
GRAHAM C. GOODWIN ${ }^{\dagger}$ AND JAMES S. WELSH ${ }^{\ddagger}$
}

\begin{abstract}
It is well known in system identification that, under output feedback control without persistent excitation, the resultant estimate of the process will be biased. In non-parametric identification the estimate is biased towards the negative inverse of the controller. A similar relationship is shown to exist for parametric identification. Indeed, we show the existence of a fundamental sensitivity to the noise model in the parametric case. However, in the parametric case, constraints are generally required, depending on the relative degree of the controller, to ensure a causal estimate. The implications of this bias to a simple adaptive control algorithm are also examined.
\end{abstract}

1. Introduction. Closed loop identification is motivated by the need to identify a process operating under output feedback. This is generally required if the process to be identified is unstable or poorly damped. Adaptive control is an area that inherently utilises closed loop identification. Industry may also demand closed loop identification experiments, for production and/or security reasons. In addition, it has been argued [Hjalmarsson, Gevers, and Bruyne, 1996] that closed loop experiments give better performance over open loop experiments when the identified model is used for control design.

It is well known in non-parametric closed loop identification, [Welsh and Goodwin, 2002], [Heath, 2001], [Ljung, 1999], [Wellstead, 1981], [Wellstead, 1977] and [Söderström and Stoica, 1989], that without an externally applied persistent excitation signal, or when the signal to noise ratio is low, then the estimate $\hat{G}$ approaches $\frac{-1}{C}$ where $\mathrm{C}$ is the controller transfer function.

On the other hand, it has recently been shown for parametric closed loop estimation, in the prediction error framework [Ljung and Forssell, 1998], that identifiability is possible even if the experiment is deemed not to be informative enough [Ljung, 1999]. It is shown that this estimate is both consistent and unbiased provided: (i) the model structure is constrained such that the noise model $\hat{H}$ is fixed equal to the true noise model $H_{o}$, (ii) the model structure $\hat{G}$ contains the true system $G_{o}$ and (iii) $\hat{G}$ is constrained such that $\hat{G} C$ has relative degree of at least 1. Under these constraints, it is shown that $\hat{G}$ approaches $G_{o}$ for large data length. Specifically, this is formulated using the plant input data spectrum and spectral factorisation.

\footnotetext{
* Received on February 11, 2002; accepted for publication on September 20, 2002.

${ }^{\dagger}$ Graham C. Goodwin is with the Department of Electrical and Computer Engineering., University of Newcastle, Callaghan, NSW Australia, 2308 Tel +61 24921 7072. Fax +6124960 1712. E-mail: eegcg@ee.newcastle.edu.au

${ }^{\ddagger}$ James S. Welsh is with the Department of Electrical and Computer Engineering, University of Newcastle, Callaghan, NSW Australia, 2308. E-mail: jwelsh@ee.newcastle.edu.au
} 
In this paper we consider discrete systems. We approach the parametric problem using well known optimisation techniques [Morari and Zafiriou, 1989] and [Goodwin, Graebe, and Salgado, 2000]. By considering the reference input to be zero, we first verify the result of [Ljung and Forssell, 1998] albeit via a slightly different proof technique. By the same methodology, we show that $\hat{G}=\frac{-1}{C}$ if we do not impose a constraint on the relative degree of $\hat{G} C$. This aligns with the non-parametric case. As well, we show that for undermodelling of the true noise model, we again obtain a $\hat{G}$ which is biased towards $\frac{-1}{C}$. As $\hat{G}$ is biased towards $\frac{-1}{C}$ we note that in the parametric case this could well result in a non-causal estimate, which, is of no consequence in the non-parametric case. We take this into account by adding a constraint on the optimisation to ensure our estimate has a relative degree which is not less than that of the true plant. Early versions of the work presented here have also appeared in [Welsh and Goodwin, 2001] and [Goodwin and Welsh, 2002].

Turning to the implications of these results for Adaptive Control, we recall early work [Astrom and Wittenmark, 1973] on controlling a system with constant but unknown parameters which showed, inter-alia, that combining a least squares parameter estimator with minimum variance control will yield the correct controller parameters if the parameters converge. There has been a large amount of follow-up interest in Adaptive Control which falls under a number of different headings including, but not limited to, Self Tuning [Astrom, 1983] [Sastry and Bodson, 1989], Automatic Tuning [Astrom and Hagglund, 1995], Iterative Feedback Tuning [Hjalmarsson, Gevers, and Lequin, 1998], the Windsurfer Approach [Lee, Anderson, Kosut, and Mareels, 1993] and the Zang Scheme [Zang, Bitmead, and Gevers, 1991]. In addition, it has been argued [Hjalmarsson, Gevers, and Bruyne, 1996] that closed loop experiments give better performance over open loop experiments when the identified model is used for control design. Closed loop issues of identification and control have also been surveyed [Van den Hof and Schrama, 1995] where a number of the iterative schemes mentioned above were evaluated. These works emphasise the link between the closed loop experiment and the resulting closed loop performance.

Here we take a different view and investigate the effect of disturbances on the resulting closed loop performance. For simplicity, we treat single input single output discrete time systems and use an adaptive tuning algorithm with separation of identification and control, i.e. we use an indirect algorithm. To highlight the issues associated with disturbances, we examine an extreme case where the reference signal is zero. In the sequel we refer to this as "self excited". We appreciate that this is an extreme case which will probably not be met in practice. Nonetheless, much of the literature on adaptive control does not exclude this possibility [Goodwin and Sin, 1984]. Thus, the results in the current paper serve to reinforce the notion that external reference signals are highly desirable, if not essential in the context of self tuning or adaptive control. 
Specifically, we show that when the disturbances are correctly modelled and the controller has sufficient complexity, then there exists a unique stationary point for the control system parameters. In a sense this is not surprising since the early work of [Astrom and Wittenmark, 1973], together with subsequent work on stochastic adaptive control show the convergence of the output to minimum variance control. Note however that this work does not claim that the system coefficients are correct and indeed in general they are not.

A key tool used here to analyse this problem is the parametric bias expression for the estimate achieved with undermodelling of the disturbance in closed loop parametric identification. This shows that the estimate is inherently a function of the controller.

Under adaptive tuning conditions, the controller is, in turn, a function of the estimates. This suggests that one should analyse this as a set of simultaneous equations so as to study the stationary points of adaptive control algorithms.

In the early 80's it was shown that in several adaptive control algorithms, instability could result due to unmodelled sinusoidal disturbances [Rhors, Valvani, Athans, and Stein, 1982] [Rhors, Valvani, Athans, and Stein, 1985]. These results have subsequently become known as the Rhors examples. These examples highlight a large sensitivity to unmodelled noise dynamics. The result in the current paper is related to that of Rhors since we are also examining sensitivity to disturbances. The difference here is that we consider stationary stochastic disturbances where the undermodelling can be varied.

The layout of the remainder of the paper is as follows. In Section 2 we define the notation and the closed loop system considered in this paper. We determine expressions for the bias, in parametric closed loop identification, when no external reference is applied in Section 3. In Section 4 we examine the implications of this bias on a simple self excited adaptive control algorithm. Section 5 draws conclusions.

2. Preliminaries. Consider the closed loop system shown in figure 1.

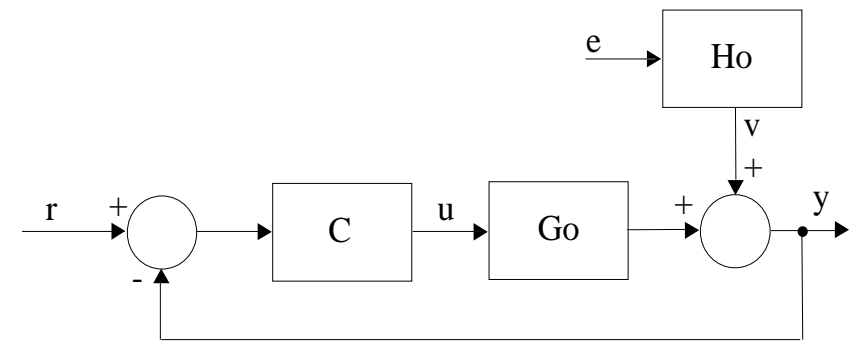

FIG. 1. Closed Loop System

Here the true system output is given by

$$
y(t)=G_{o} u(t)+H_{o} e(t)
$$


where $e$ is assumed to be stationary gaussian white noise.

The system to be identified is also constrained to be of the same structure as equation (1), with the estimates of the system model components being denoted by $\hat{G}$ and $\hat{H}$ respectively.

The parameters of $\hat{G}$ are estimated by using a Maximum Likelihood estimator. This leads to the estimate:

$$
\begin{aligned}
\hat{\theta} & =\underset{\theta}{\arg \min } J(\theta, u, y) \\
J(\theta, u, y) & =\frac{1}{N} \sum_{t=1}^{N} \epsilon^{T}(t, \theta) \epsilon(t, \theta) \\
\epsilon(t, \theta) & =y(t)-\hat{y}(t \mid \theta)=\frac{1}{\hat{H}}(y(t)-\hat{G} u(t))
\end{aligned}
$$

where $\mathrm{N}$ is the data length. (Note that this is, in fact the conditional maximum likelihood solution and it is implicitly assumed that the plant and noise model initial conditions are zero.)

We now substitute equation (1) into equation (4) to obtain

$$
\epsilon(t, \theta)=\frac{1}{\hat{H}}\left(G_{o} u(t)+H_{o} e(t)-\hat{G} u(t)\right) .
$$

Using the output sensitivity function,

$$
S_{o}=\frac{1}{1+G_{o} C}
$$

we can express the process input as

$$
u(t)=C S_{o} r(t)-C S_{o} H_{o} e(t) .
$$

Then substituting equation (7) into (5) we have

$$
\epsilon(t, \theta)=\frac{1}{\hat{H}}\left(-\delta G C S_{o} r(t)+\delta G C S_{o} H_{o} e(t)+H_{o} e(t)\right)
$$

where $\delta G=\hat{G}-G_{o}$.

Finally re-arranging (8) we obtain

$$
\epsilon(t, \theta)=\frac{1}{\hat{H}} C S_{o}\left(G_{o}-\hat{G}\right) r(t)+S_{o}(1+\hat{G} C) \frac{H_{o}}{\hat{H}} e(t) .
$$

To formulate the optimisation problem, we make the following definitions and assumptions. We define $S_{c}$ to be the set of causal transfer functions and $S_{c}^{\perp}$ to be its complement, i.e. the set of non-causal transfer functions. Now assuming $G_{o} C \in S_{c}$, we then require that $\hat{G} C \in S_{c}$. As we are considering the case of zero signal to noise ratio we set the reference input, $r$, to be zero. This gives

$$
\epsilon(t, \theta)=S_{o}(1+\hat{G} C) \frac{H_{o}}{\hat{H}} e(t) .
$$


We proceed by considering the discrete system in the frequency domain. Via Parseval's theorem [Ljung, 1999], the asymptotic cost function takes the form

$$
J=\underset{\hat{G} C \in S_{c}}{\arg \min }\left\|\frac{1+\hat{G}\left(e^{j \omega}\right) C\left(e^{j \omega}\right)}{\left(1+G_{o}\left(e^{j \omega}\right) C\left(e^{j \omega}\right)\right) \Lambda\left(e^{j \omega}\right)}\right\|_{2}^{2}
$$

where

$$
\Lambda\left(e^{j \omega}\right)=\frac{\hat{H}\left(e^{j \omega}\right)}{H_{o}\left(e^{j \omega}\right)} .
$$

In the sequel we will fix $\hat{H}$. In particular, we are interested in sensitivity to the noise model and thus $\hat{H}$ will not necessarily be taken as $H_{o}$. Also we assume that $\hat{G}$ and $\hat{H}$ are independently parameterised.

3. Bias in Parametric Closed Loop Identification. Here we examine 2 cases. In Case A, we consider having perfect knowledge of the noise model, i.e $\hat{H}=$ $H_{o}$. Here we establish the result of [Ljung and Forssell, 1998]. Case B examines the sensitivity of the estimate to undermodelling of the noise model. We first show that, for an unconstrained estimate, we obtain the same result as in the non-parametric case i.e $\hat{G}=\frac{-1}{C}$. We then consider when the relative degree of $\hat{G}$ is constrained to be not less then that of $G_{o}$. In this case, $\hat{G}$ turns out to be biased toward $\frac{-1}{C}$ by an amount depending on the extent of the noise model error. Simulations are then presented to validate our results.

3.1. Case A: Consummate Noise Model. The following result re-establishes the result presented in [Ljung and Forssell, 1998].

Lemma 1. Under the conditions of a perfectly known noise model and when $\hat{G} C$ is constrained to have relative degree of not less than 1, then the optimisation in equation (11) yields the result $\hat{G}=G_{o}$.

Proof. Under the conditions of a perfect noise model we have

$$
\Lambda\left(e^{j \omega}\right)=\frac{\hat{H}\left(e^{j \omega}\right)}{H_{o}\left(e^{j \omega}\right)}=1 .
$$

We separate equation (11) into causal and non-causal components.

$$
J=\underset{\hat{G} C \in S_{c}}{\arg \min }\left\|1+\frac{\hat{G}\left(e^{j \omega}\right) C\left(e^{j \omega}\right)-G_{o}\left(e^{j \omega}\right) C\left(e^{j \omega}\right)}{1+G_{o}\left(e^{j \omega}\right) C\left(e^{j \omega}\right)}\right\|_{2}^{2} .
$$

For simplicity we define $\mathbf{F}\left(e^{j \omega}\right) \in S_{c}$ such that

$$
\mathbf{F}\left(e^{j \omega}\right)=\frac{\hat{G}\left(e^{j \omega}\right) C\left(e^{j \omega}\right)-G_{o}\left(e^{j \omega}\right) C\left(e^{j \omega}\right)}{1+G_{o}\left(e^{j \omega}\right) C\left(e^{j \omega}\right)}
$$

then

$$
\left\|1+\mathbf{F}\left(e^{j \omega}\right)\right\|_{2}^{2}=\|1\|_{2}^{2}+\left\|\mathbf{F}\left(e^{j \omega}\right)\right\|_{2}^{2}+\frac{1}{2 \pi} \int_{-\pi}^{\pi} 2 \mathcal{R} e\left[\mathbf{F}\left(e^{j \omega}\right)\right] d \omega .
$$


The third term on the right hand side can be easily shown [Churchill and Brown, 1990] to be zero for a strictly proper analytic function, $F(z)$, i.e.

$$
\begin{aligned}
\mathcal{R} e\left[\frac{1}{\pi} \int_{-\pi}^{\pi} \mathbf{F}\left(e^{j \omega}\right) d \omega\right] & =\frac{1}{\pi} \mathcal{R} e\left[\frac{1}{j} \oint_{c} \frac{\mathbf{F}\left(z^{-1}\right)}{z} d z\right] \\
& =0 .
\end{aligned}
$$

We thus see that we can express the cost function as,

$$
J=1+\underset{\hat{G} C \in S_{c}}{\arg \min }\left\|\frac{\hat{G}\left(e^{j \omega}\right) C\left(e^{j \omega}\right)-G_{o}\left(e^{j \omega}\right) C\left(e^{j \omega}\right)}{1+G_{o}\left(e^{j \omega}\right) C\left(e^{j \omega}\right)}\right\|_{2}^{2} .
$$

From which it can be seen that the minimum occurs when $\hat{G}=G_{o}$.

The above result confirms that of [Ljung and Forssell, 1998] where the same conclusion was established using spectral factorisation.

3.2. Case B: Undermodelling in the Noise Model. We first show that, with no constraints on the relative degree the estimate, $\hat{G}$, is the same as the nonparametric case irrespective of having the true noise model.

Lemma 2. With no constraints on the relative degree of $\hat{G} C$ and independent of the accuracy of the noise model, the estimate is given by $\hat{G}=\frac{-1}{C}$.

Proof. The result is immediate by removing the constraint, $\hat{G} C \in S_{c}$, from equation (11).

Next the sensitivity of the self excited, parametric estimate, to the noise model will be examined when constraints on the relative degree of $\hat{G}$ are added. In particular, we show that with noise undermodelling, the estimate $\hat{G}$ becomes biased towards the negative inverse of the controller as is the case with the non-parametric estimate.

It is clear from the non-parametric case where $\hat{G}$ approaches $\frac{-1}{C}$, that if a similar relationship holds for the parametric estimate, then an unconstrained estimate may be non-causal depending on the relative degree of $C$. We therefore need to constrain the estimate such that the appropriate relative degree is imposed as a constraint on the class of allowable estimates $\hat{G}$.

In the sequel, we assume that the relative degree of $C$ is $d_{c}$, the relative degree of $G_{o}$ is $d_{g}$ and that $d_{c} d_{g} \geq 1$.

We begin with some further definitions to make the future discussion clearer.

The inverse of the noise undermodelling is $\Lambda^{-1}\left(z^{-1}\right)$. This can be expanded as

$$
\begin{aligned}
\Lambda^{-1}\left(z^{-1}\right) & =1+\sum_{k=1}^{\infty} \lambda_{k}^{i} z^{-k} \\
& =\bar{\Lambda}+z^{-d} \grave{\Lambda}
\end{aligned}
$$


where $d$ is the combined relative degree of $G_{o} C$ i.e. $d=d_{g}+d_{c}, \lambda_{k}^{i}$ are the coefficients (actually Markov parameters) of $\Lambda^{-1}$ and

$$
\begin{aligned}
& \bar{\Lambda}=1+\sum_{k=1}^{d-1} \lambda_{k}^{i} z^{-k}, \\
& \grave{\Lambda}=\sum_{k=d}^{\infty} \lambda_{k}^{i} z^{-k+d} .
\end{aligned}
$$

$\bar{\Lambda}$ combines the first $d-1$ Markov parameters of $\Lambda^{-1}$.

We then have

THEOREM 3. With noise undermodelling described by:

$$
\Lambda(z)=\frac{\hat{H}(z)}{H_{o}(z)}
$$

where $\Lambda(z)$ is bi-proper. If we constrain the allowable class of estimates such that $\hat{G}$ has (at least) the same relative degree as $G_{o}$, then the constrained estimate $\hat{G}(z)$ takes the asymptotic value:

$$
\hat{G}_{a}(z)=\Lambda(z) \bar{\Lambda}(z) G_{o}(z)+(1-\Lambda(z) \bar{\Lambda}(z))\left(-C(z)^{-1}\right)
$$

where $\bar{\Lambda}$ is defined in equation (21).

Proof. By hypothesis the undermodelling of the true noise is described by $\Lambda\left(z^{-1}\right) \ngtr 1$.

The asymptotic cost function is then as in (11), i.e.

$$
J=\underset{\hat{G} C \in S_{c}}{\arg \min }\left\|\frac{1+\hat{G}(z) C(z)}{\left(1+G_{o}(z) C(z)\right) \Lambda(z)}\right\|_{2}^{2} .
$$

Constraining $\hat{G} C$ to have relative degree $d$, equation (25) can be expressed as

$$
J=\underset{z^{-d} \hat{F} \in S_{c}}{\arg \min }\left\|\frac{1+z^{-d} \hat{F}(z)}{\left(1+z^{-d} F_{o}(z)\right) \Lambda(z)}\right\|_{2}^{2}
$$

where

$$
\begin{aligned}
z^{-d} \hat{F}(z) & =\hat{G}(z) C(z) \\
z^{-d} F_{o}(z) & =G_{o}(z) C(z) \\
d & =\left\{G_{o}(z) C(z)\right\}_{r d e g} .
\end{aligned}
$$

and $\{.\}_{r d e g}$ is the relative degree operator.

To aid the clarity of the following development we will suppress the function argument. Until otherwise noted all functions belong to the discrete frequency domain, $z$. 
Rearranging equation (26) we have

$$
\begin{aligned}
J & =\underset{z^{-d} \hat{F} \in S_{c}}{\arg \min }\left\|\frac{\Lambda^{-1}+\Lambda^{-1} z^{-d} \hat{F}}{1+z^{-d} F_{o}}\right\|_{2}^{2} \\
& =\underset{z^{-d} \hat{F} \in S_{c}}{\arg \min }\left\|\frac{\bar{\Lambda}+z^{-d} \grave{\Lambda}}{1+z^{-d} F_{o}}+\frac{\Lambda^{-1} z^{-d} \hat{F}}{1+z^{-d} F_{o}}\right\|_{2}^{2} \\
= & \underset{z^{-d} \hat{F} \in S_{c}}{\arg \min }\left\|\bar{\Lambda}+\frac{z^{-d}\left(\grave{\Lambda}-\bar{\Lambda} F_{o}+\Lambda^{-1} \hat{F}\right)}{1+z^{-d} F_{o}}\right\|_{2}^{2} \\
= & \underset{z^{-d} \hat{F} \in S_{c}}{\arg \min }\left\|\bar{\Lambda}+z^{-d} \mathbf{F}\right\|_{2}^{2}
\end{aligned}
$$

where

$$
\mathbf{F}=\frac{\grave{\Lambda}-\bar{\Lambda} F_{o}+\Lambda^{-1} \hat{F}}{1+z^{-d} F_{o}}
$$

then

$$
\left\|\bar{\Lambda}+z^{-d} \mathbf{F}\right\|_{2}^{2}=\|\bar{\Lambda}\|_{2}^{2}+\left\|z^{-d} \mathbf{F}\right\|_{2}^{2}+\frac{1}{\pi} \mathcal{R} e\left[\frac{1}{j} \oint_{c} \bar{\Lambda}^{*} z^{-d} \mathbf{F} \frac{d z}{z}\right] .
$$

Now, from [Churchill and Brown, 1990],

$$
\frac{1}{\pi} \mathcal{R} e\left[\frac{1}{j} \oint_{c} \bar{\Lambda}^{*} z^{-d} \mathbf{F} \frac{d z}{z}\right]=0
$$

since

$$
\begin{aligned}
<z^{-n}, z^{-m}> & =\frac{1}{2 \pi} \oint_{c} z^{n} z^{-m} \frac{d z}{z} \\
& =\frac{1}{2 \pi} \oint_{c} \frac{d z}{z^{m-n+1}} \\
& =0 \quad \text { for }(m-n+1) \geq 2 .
\end{aligned}
$$

From equations (34) and (35) we see the constrained minimum occurs when

$$
z^{-d} \Lambda^{-1} \hat{F}=z^{-d} \bar{\Lambda} F_{o}-z^{-d} \grave{\Lambda} .
$$

Substituting $G_{o}, \hat{G}$ and $C$ into equation (40) and solving for $\hat{G}$ yields the result

$$
\begin{aligned}
\hat{G}_{a} & =\Lambda \bar{\Lambda} G_{o}+z^{-d} \Lambda \grave{\Lambda}\left(\frac{-1}{C}\right) \\
& =\Lambda \bar{\Lambda} G_{o}+(1-\Lambda \bar{\Lambda})\left(\frac{-1}{C}\right) .
\end{aligned}
$$

Remark 4. Notice $\hat{G}_{a}$ has a relative degree, greater than or equal to, $G_{o}$. Also, we see that $\hat{G}_{a}(z)$ lies between $G_{o}(z)$ and $-C(z)^{-1}$ where the displacement towards $-C(z)^{-1}$ depends on the extent of the undermodelling.

Some special cases of the result are presented below:

Corollary 5. 
$i$. For the case where the $\left\{G_{o}(z) C(z)\right\}_{r d e g}=1$, then $\bar{\Lambda}=1$ and the result can be expressed more simply as

$$
\hat{G}_{a}(z)=\Lambda(z) G_{o}(z)+(1-\Lambda(z))\left(\frac{-1}{C(z)}\right) .
$$

ii. Under the same conditions as in part (i), if in addition, the noise undermodelling is described by a single pole, i.e. $\Lambda(z)=\frac{z-\alpha}{z}$, the expression for $\hat{G}(z)$ becomes:

$$
\hat{G}_{a}(z)=\left(1-\alpha z^{-1}\right) G_{o}(z)+\alpha z^{-1}\left(-C(z)^{-1}\right) .
$$

iii. Under the same conditions as in part (ii), if $\alpha \rightarrow 1$ and fast sampling is used, then we have that

$$
\hat{G}_{a}(z) \approx-C(z)^{-1}
$$

for $z=e^{j \omega}$ and $\omega$ less than the folding frequency.

Proof.

i. Immediate from Theorem 3.

ii. Immediate from Theorem 3.

iii. For $\alpha \rightarrow 1$, we have

$$
\hat{G}_{a}(z) \approx G_{o}(z)\left(1-z^{-1}\right)-z^{-1} C(z)^{-1} .
$$

Now the first term in equation (46) is the difference between the impulse response of $G_{o}(z)$ and the impulse response shifted by one sample. Hence with fast sampling, the difference converges to zero. The second term then yields

$$
\hat{G}_{a}(z) \approx \frac{-1}{z C(z)}
$$

This corresponds to the impulse response of $\frac{-1}{C}$ shifted by one sample. Again with fast sampling, we have

$$
\hat{G}_{a}(z) \approx \frac{-1}{C(z)}
$$

3.3. Simulation. To demonstrate the sensitivity of the estimate to that of the noise model (as shown above) we performed simulations in MATLAB where we considered a single pole, $\left(\frac{z}{z-\alpha}\right)$, to exist in the true noise model but not in the hypothetical noise model. We varied the position of this pole by adjusting $\alpha$ between 0 and 0.999 . When $\alpha=0$ we have a consummate noise model. 
The noise was generated using a maximal length pseudo random binary sequence (PRBS) using a shift register having 11 bits. The mean value was then shifted to zero. This gave an autocorrelation of exactly 1 at zero lag and 0 for all other lags.

The closed loop system was constructed as shown in figure 1 . The controller was taken as a unity gain. The process consisted of a finite impulse response (FIR) model of the continuous time process $\frac{1}{s+1}$ sampled at $\Delta=0.1$ and truncating after a number of samples, $n+1$, to obtain the desired order $n$.

Example 1. The first system we simulated uses a FIR model of order 12. To demonstrate the effect of the relative degree constraint we add a pure delay of 3 samples $\left(z^{-3}\right)$ to the controller, giving it a relative degree of 3. Undermodelling in the noise model was applied using $\alpha=0.999$. The Bode diagram shown in figure 2 shows that Theorem 3 predicts the estimate with a high degree of accuracy.
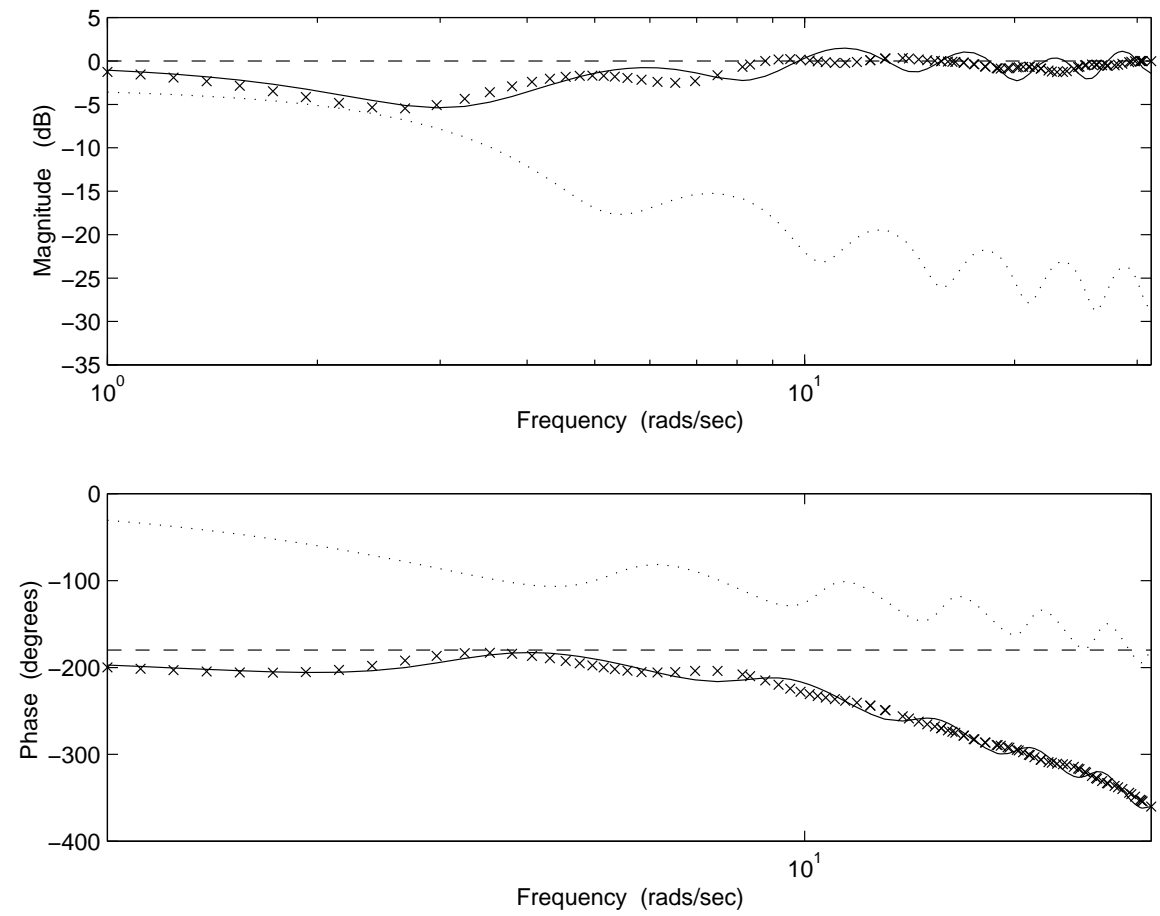

FIG. 2. Bode Plots Using PRBS. dot: $G_{o}$, dash: $\frac{-1}{C}$, solid: $\hat{G}$ and $x: \hat{G}_{a}$.

Example 2. The following simulations use a finite impulse response model of order 6 , namely $G_{o}(z)=0.0952 z^{-1}+0.0861 z^{-2}+0.0779 z^{-3}+0.0705 z^{-4}+0.0638 z^{-5}+$ $0.0577 z^{-6}$, with no extra delays added to the system. The results of these simulations are presented as Bode diagrams in figure 3 for a number of values of $\alpha$ as tabulated in table 1. It is seen that, when the noise model is known perfectly, we obtain a near perfect estimate of the process despite having no externally applied reference signal. 
This is in accord with Lemma 1. If however, we undermodel the noise system we see from figure 3 , that the process estimate $\hat{G}$ approaches $\frac{-1}{C}$, which is as predicted by Corollary 5 (iii).
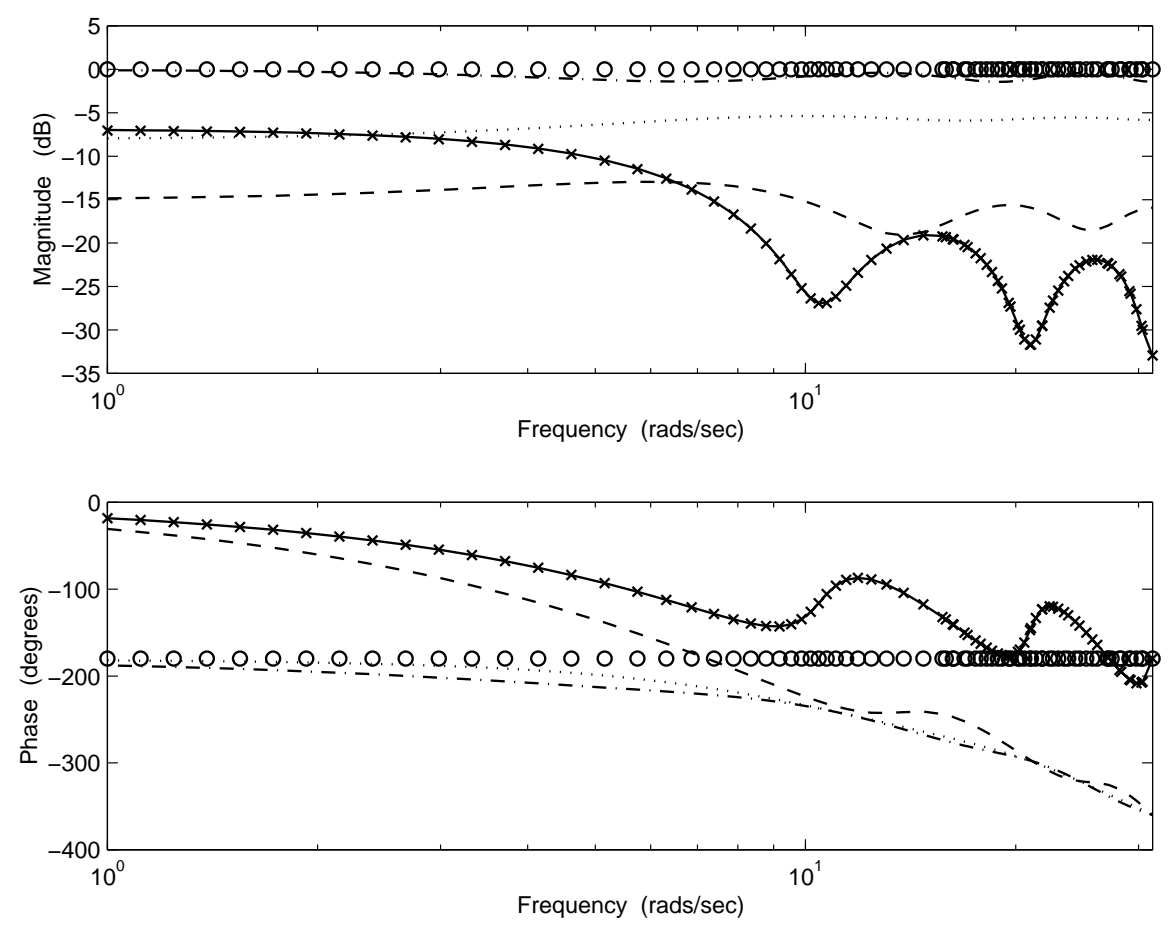

FIG. 3. Bode Plots Using PRBS. x: Go, o: $\frac{-1}{C}$, solid: $\alpha=0$, dashed: $\alpha=0.1998$, dot: $\alpha=0.5994$ and dash-dot: $\alpha=0.999$.

Indeed, by application of Corollary 5, we can calculate the exact value of our estimated process for the various values of $\alpha$. The result given by equation 44 is given as $\hat{G}_{a}$ in table 1 where it is compared with the experimental estimates, $\hat{G}$. We see that there is near perfect correspondence between $\hat{G}$ and $\hat{G}_{a}$ as anticipated.

Note that for small $\alpha, \hat{G}$ and $\hat{G}_{a}$ depart significantly from $G_{o}$ showing the strong sensitivity to the noise model.

As further evidence of the strong sensitivity to the noise model, we note that during initial simulations it was found that the exact value of the process could not be estimated even with a perfect noise model. These simulations were carried out using the MATLAB random noise generator. When the autocorrelation of the output signal from this source was examined it was seen to have values which differed slightly from zero for all lags. The results using this noise source are shown in the Bode diagram of figure 4 . This suggests that it would be impossible in any practical sense to estimate $G_{o}$ without having a strong reference signal. 
TABle 1

Measured and Calculated Estimates

\begin{tabular}{|c||c|c|c|c|c|c|c|c|}
\hline$\alpha$ & Model & 1 & $z^{-1}$ & $z^{-2}$ & $z^{-3}$ & $z^{-4}$ & $z^{-5}$ & $z^{-6}$ \\
\hline 0 & $\hat{G}$ & 0 & 0.0952 & 0.0861 & 0.0779 & 0.0705 & 0.0638 & 0.0577 \\
\hline & $\hat{G}_{a}$ & 0 & 0.0956 & 0.0866 & 0.0784 & 0.0710 & 0.0641 & 0.0582 \\
\hline 0.1998 & $\hat{G}$ & 0 & -0.1046 & 0.0671 & 0.0607 & 0.0549 & 0.0497 & 0.0450 \\
\hline & $\hat{G}_{a}$ & 0 & -0.1037 & 0.0681 & 0.0618 & 0.0560 & 0.0508 & 0.0443 \\
\hline 0.5994 & $\hat{G}^{2}$ & 0 & -0.5042 & 0.0291 & 0.0263 & 0.0238 & 0.0215 & 0.0195 \\
\hline & $\hat{G}_{a}$ & 0 & -0.5037 & 0.0301 & 0.0274 & 0.0250 & 0.0227 & 0.0026 \\
\hline 0.9990 & $\hat{G}$ & 0 & -0.9038 & -0.0090 & -0.0081 & -0.0073 & -0.0066 & -0.0060 \\
\hline & $\hat{G}_{a}$ & 0 & -0.9058 & -0.0091 & -0.0084 & -0.0076 & -0.0071 & -0.0571 \\
\hline
\end{tabular}
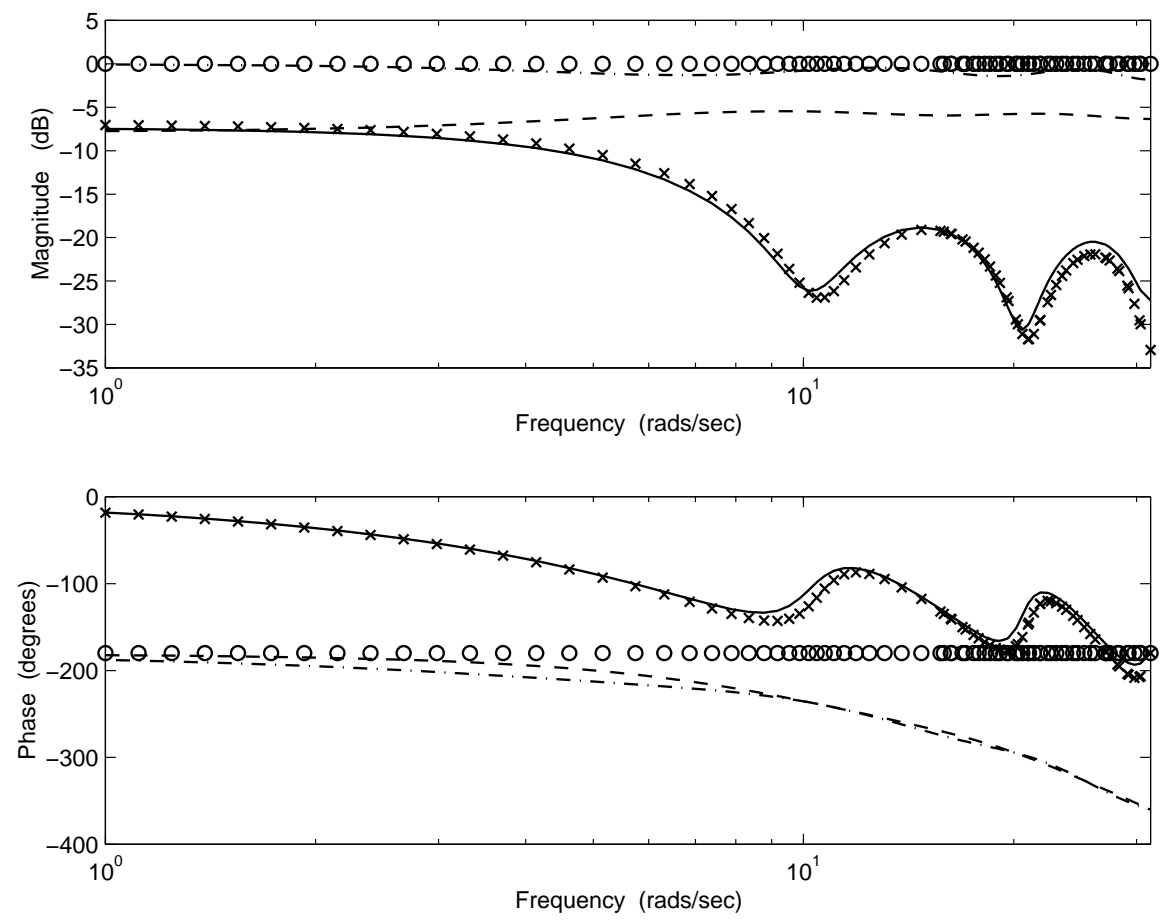

FIG. 4. Bode Plots Using MATLAB Random Number Generator. $x: G_{o}$, o: $\frac{-1}{C}$, solid: $\alpha=0$, dashed: $\alpha=0.5994$ and dash-dot: $\alpha=0.999$. 
4. Implications in Adaptive Control. We next examine the implications of the result presented above, on a simple adaptive control algorithm which relies only on the disturbance for excitation. We call this "self excited" adaptive control.

A simple indirect adaptive tuning procedure consists of a recursion which is repeated until a particular performance measure has been satisfied. This procedure is performed under output feedback control and consists of the following steps:

i. Set $i=1$, and choose an initial stabilising controller $C_{0}$.

ii. Collect closed loop data using the controller $C_{i-1}$ and estimate the model $\left(\hat{G}_{i}\right)$.

iii. Design a new controller $\left(C_{i}\right)$ using the estimate $\left(\hat{G}_{i}\right)$ from (ii).

iv. Set $i=i+1$ and repeat (ii) and (iii) until the required performance measure is achieved.

Examining this algorithm, we see that the controller $\left(C_{i}\right)$ designed at iteration $i$ is a function of the estimate at the same iteration $\left(\hat{G}_{i}\right)$. From equation $(24)$, we see that $\hat{G}_{i}$ is a function of $G_{o}$ and $C_{i-1}$. Thus $\hat{G}_{i}$ is a function of $\hat{G}_{i-1}$. The relationship takes the form of a forced nonlinear iteration in function space.

The above description gives the essential features of the adaptive control algorithm we will study here. In the next section, we will examine the stationary points of the iteration in function space (assuming they exist) for the above iteration when used with a specific control system design procedure.

4.1. Main Results. To detail the specific adaptive control algorithm of interest here, we begin with the controller design methodology. In principle, we could choose any appropriate control system design strategy. However, to give maximal insight, we choose the simplest possible method namely affine parameterisation design for stable linear systems [Goodwin, Graebe, and Salgado, 2000]. Specifically, to design the controller we begin by parameterising it as

$$
C(z)=\frac{Q(z)}{1-\hat{G}(z) Q(z)} .
$$

Here $Q(z)=F(z) \hat{G}_{i n v}(z)$ where $\hat{G}_{i n v}(z)$ is equal to $\hat{G}(z)^{-1}$ when $\hat{G}(z)$ is minimum phase and otherwise reflects the non-minimum phase zeros through the unit circle. The filter $F(z)$ is chosen to yield a proper $Q(z)$.

Assuming the initial closed loop system is stabilised by an appropriate controller, the direct method of identification is applied. Here we collect measurements of the input $u(t)$ and output $y(t)$ of the process then employ a maximum likelihood estimator to obtain a set of parameters representative of the process. Note that the likelihood function is a nonlinear function of the parameters in a rational model and thus we use a standard iterative procedure to maximise the likelihood at each step of the algorithm. Based on the estimate so obtained we use (49) to design the controller for the next iteration. 
We begin the analysis of the sensitivity associated with this algorithm by presenting the following result which embellishes the iteration described above.

Lemma 6 . The evolution of the estimate $\hat{G}$ under adaptive control is in the space of transfer functions and can be expressed as

$$
\hat{G}_{i+1}(z)=\Lambda(z) G_{o}(z)-(1-\Lambda(z)) f\left(\hat{G}_{i}(z)\right)^{-1} .
$$

where $f($.$) represents the relationship between \hat{G}_{i}$ and $C_{i}$.

Proof. From (43) we have

$$
\hat{G}(z)=\Lambda(z) G_{o}(z)+(1-\Lambda(z))\left(-C(z)^{-1}\right) .
$$

Let $\hat{G}_{i}$ denote the estimate of $G$ at the $i^{t h}$ iteration. Equation (51) then becomes

$$
\hat{G}_{i+1}(z)=\Lambda(z) G_{o}(z)+(1-\Lambda(z))\left(-C_{i}(z)^{-1}\right) .
$$

where $C_{i}$ is a function of $\hat{G}_{i}$ which we express as $f\left(\hat{G}_{i}(z)\right)$.

The result then follows.

Lemma 6 captures the essence of the problem exclusively. However, to gain additional insight, we make the simplifying assumption that the system is stable, minimum phase and has relative degree one. Thus, we assume that at each step in the iteration we can simply choose $Q_{i}(z)=\hat{G}_{i}(z)^{-1} F(z)$ where $F(z)=\frac{1-\beta}{z-\beta}$.

THEOREM 7. With adaptive control under the assumptions above then, if convergence to a unique stationary point occurs, the stationary point, $\hat{G}_{s p}(z)$, of the estimate $\hat{G}$ must be:

$$
\hat{G}_{s p}(z)=\frac{\Lambda(z) G_{o}(z) F(z)}{1-\Lambda(z)(1-F(z))}
$$

and the corresponding stationary point for the controller must be:

$$
C_{s p}(z)=\frac{1-\Lambda(z)(1-F(z))}{\Lambda(z) G_{o}(z)(1-F(z))} .
$$

Proof. Equation (49) can be written directly in terms of the filter transfer function $F(z)$ as

$$
C(z)=\frac{F(z) \hat{G}(z)^{-1}}{1-F(z)}
$$

Solving (51) and (55) simultaneously we obtain the result.

REMARK 8. Note that there is a potential in the algorithm for obtaining estimates of increasing degree. However, this does not happen in the relative degree one case.

The next question of interest is whether or not convergence to $\left(\hat{G}_{s p}, C_{s p}\right)$ actually occurs. A sufficient condition for this convergence is given in the following theorem. 
TheOREM 9. A sufficient condition for convergence of $\left(\hat{G}_{i}, C_{i}\right)$ to $\left(\hat{G}_{s p}, C_{s p}\right)$ is that

$$
\|\gamma\|_{\infty}<1
$$

where

$$
\gamma(z)=\frac{(1-\Lambda(z))(F(z)-1)}{F(z)}
$$

Proof. Substituting (55) into equation (52) gives

$$
\hat{G}_{i+1}(z)=\Lambda(z) G_{o}(z)+(1-\Lambda(z))\left(\frac{(F(z)-1) \hat{G}_{i}}{F(z)}\right) .
$$

We note that this is a linear equation of the form

$$
\hat{G}_{i+1}(z)=\gamma(z) \hat{G}_{i}(z)+\beta(z)
$$

where

$$
\begin{aligned}
& \gamma(z)=\frac{(1-\Lambda(z))(F(z)-1)}{F(z)} \\
& \beta(z)=\Lambda(z) G_{o}(z) .
\end{aligned}
$$

We write this linear equation in error form by defining

$$
\tilde{G}_{i}(z)=\hat{G}_{i}(z)-\hat{G}_{s p}(z)
$$

then equation (59) becomes

$$
\tilde{G}_{i+1}(z)=\gamma(z) \tilde{G}_{i}(z)
$$

This equation evolves in the space of transfer functions. The infinity norm of $\tilde{G}_{i}(z)$ satisfies:

$$
\left\|\tilde{G}_{n+1}\right\|_{\infty} \leq\|\gamma\|_{\infty}\left\|\tilde{G}_{n}\right\|_{\infty}
$$

Hence a sufficient condition for the iteration to be a contraction is that the $H^{\infty}$ norm of $\gamma$ be $<1$.

We next present two results which give further insight into the nature of the stationary point $\left(\hat{G}_{s p}, C_{s p}\right)$.

Lemma 10. For a stable minimum phase process, assuming a stationary point of the adaptive control algorithm exists, then the achieved closed loop is stable. Specifcally, the closed loop characteristic polynomial of the achieved system is given by

$$
A_{c l}(z)=B_{o}(z) A_{o}(z) \Omega(z) D(z)
$$


where $G_{o}(z)=\frac{B_{o}(z)}{A_{o}(z)}, F(z)=\frac{N(z)}{D(z)}$ and $\Lambda=\frac{\Gamma(z)}{\Omega(z)}$.

Proof. Using (54) the stationary point of the controller can be expressed as

$$
C_{s p}(z)=\frac{A_{o}(z)(\Omega(z) D(z)+\Gamma(z) N(z)-\Gamma(z) D(z))}{B_{o}(z) \Gamma(z)(D(z)-N(z))}
$$

where $F(z)=\frac{N(z)}{D(z)}$ and $\Lambda=\frac{\Gamma(z)}{\Omega(z)}$. The control design procedure ensures that the polynomial $D(z)$ is always chosen to be stable.

The achieved closed loop characteristic polynomial is given by

$$
A_{c l}=B_{o}(z) P(z)+A_{o}(z) L(z)
$$

where the controller is expressed as $C(z)=\frac{P(z)}{L(z)}$.

Substituting (66) into (67) yields the result.

REMARK 11. Subject to the conditions of Theorem 9, we know that $\hat{G}_{i}(z)$ will converge to $\hat{G}_{s p}(z)$. Also, Lemma 10 ensures that the final closed loop system is stable. A key point, however, is that there is no guarantee that during the iterative procedure that the true process will be stabilised at every iteration.

LEMMA 12. Assuming a stationary point exists in the adaptive control algorithm then there is a simple relationship between the final achieved sensitivity and the nominal sensitivity, namely:

$$
S(z)=S_{o}(z) S_{\Delta}(z)
$$

where

$$
S_{o}(z)=1-F(z)
$$

and

$$
S_{\Delta}(z)=\Lambda(z)
$$

where $\Lambda(z)$ is given in equation (12).

Proof. The achieved sensitivity is given by [Goodwin, Graebe, and Salgado, 2000]

$$
S(z)=S_{o}(z) S_{\Delta}(z)
$$

where

$$
S_{\Delta}(z)=\frac{1}{1+T_{s p}(z) G_{\Delta}(z)}
$$

and where $G_{\Delta}(z)$ is the relative error between the true system $G_{o}(z)=\frac{B(z)}{A(z)}$ and the estimate $\hat{G}_{s p}$. Thus,

$$
G_{\Delta}(z)=\frac{G_{o}(z)-\hat{G}_{s p}(z)}{\hat{G}_{s p}(z)} .
$$


Substituting (53) into equation (73) we obtain

$$
G_{\Delta}(z)=\frac{\Omega(z) D(z)-\Gamma(z) D(z)}{\Gamma(z) N(z)}
$$

using the notation from Lemma 10.

We can express the design sensitivity as

$$
T_{s p}=F(z) .
$$

The result follows by substituting (74) and (75) into (72).

\subsection{Simulations.}

Example 3. The process consists of a single pole system $G_{o}(z)=\frac{0.04877}{z-0.9512}$.

i. We first use an algebraic routine to find the stationary points of the adaptive control algorithm described above. Here the noise model is undermodelled by $\Lambda(z)=\frac{z-0.45}{z}$. The filter used in the controller design was $F(z)=\frac{0.9102}{z-0.08985}$. Note that for this example $\|\gamma\|_{\infty}=0.988$, thus from Theorem 9 we expect the algorithm to converge to $\left(\hat{G}_{s p}(z), C_{s p}\right)$.

Figure 5 shows the bode diagram of the true process, a number of iterations of the estimated process and the calculated stationary point using (53). It can be seen that the estimate does indeed converge to the calculated stationary point. Figure 6 shows the bode diagram of the controller at each iteration converging to the calculated stationary point.

ii. We next vary the location of the undermodelled pole in the noise model (denoted here as $\alpha$ ). We calculate the stationary point for the estimate of the process and the controller as shown in Figure 7. The differing line type represents the varying alpha. It can be seen from figure 7 that the stationary points vary considerably with alpha. The results show that we can obtain very poor estimates of the true system even with relatively small noise undermodelling.

iii. We next demonstrate the algorithm diverging. We take $\alpha$ as in part (i) but have chosen a filter $F(z)=\frac{0.3297}{z-0.6703}$ such that Lemma 10 is not satisfied. In fact in this example, $\|\gamma\|_{\infty}=2.7$. Figure 8 shows the estimate of the process diverging. Note that this is not unexpected since the sufficient conditions of Lemma 10 are not satisfied.

This result shows that even with very mild undermodelling, divergence of the adaptive control algorithm can occur depending on the choice of the design parameters used in the control law.

Example 4. Here we highlight Remark 11 by demonstrating that although the final closed loop system is stable, it is possible to have an unstable closed loop during the iterative procedure. In this example we have chosen a value of $\alpha=0.6$. Figure 9 

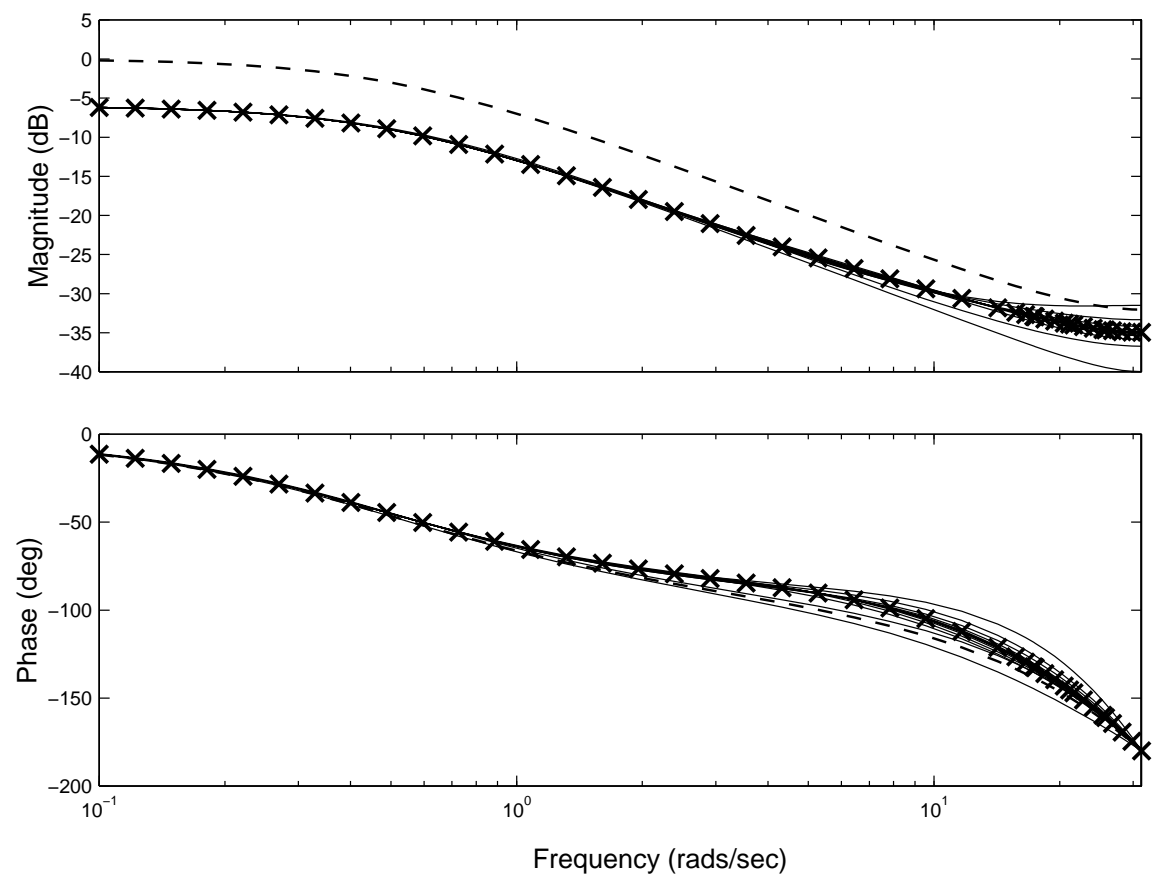

FIG. 5. Bode Plot of True Process (dash), Calculated Stationary Point (x) and Estimates (solid).
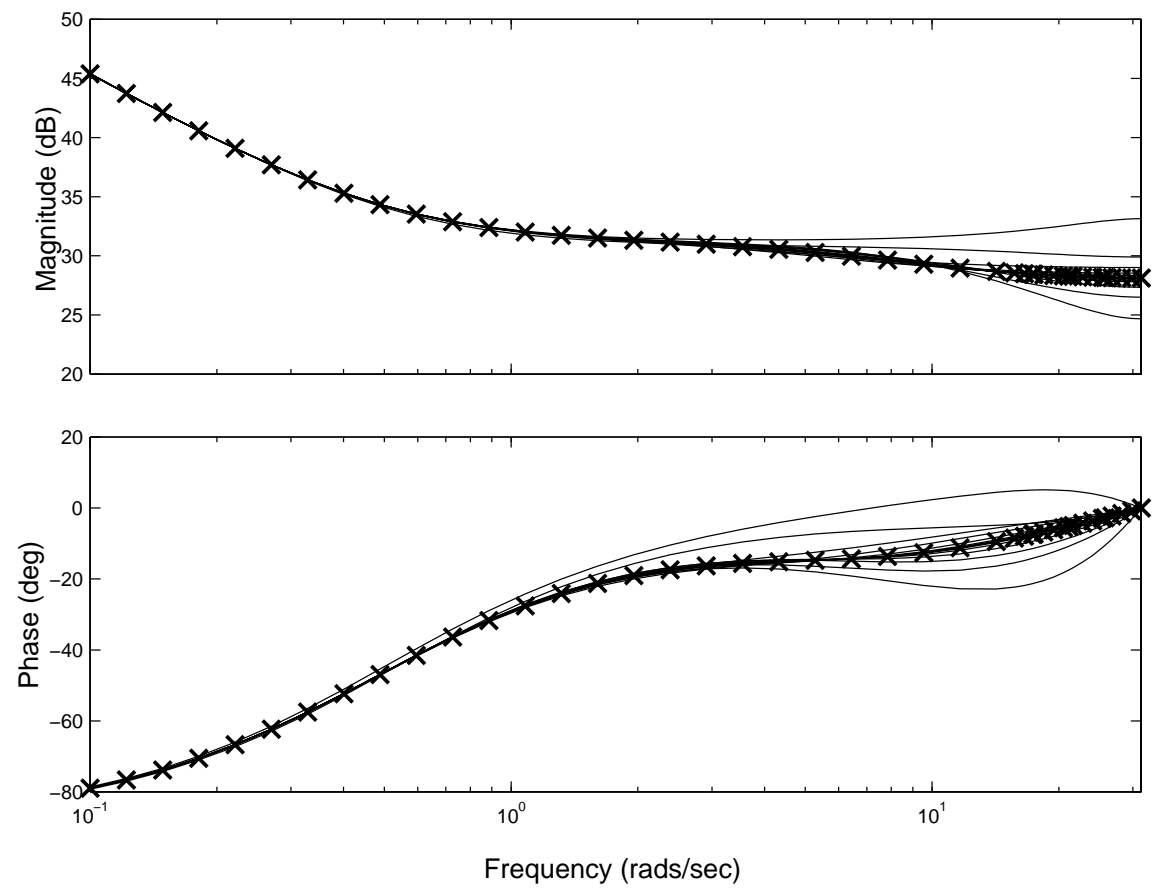

FIG. 6. Bode Plot of the Controller Stationary Point (x) and Design at each Iteration (solid). 

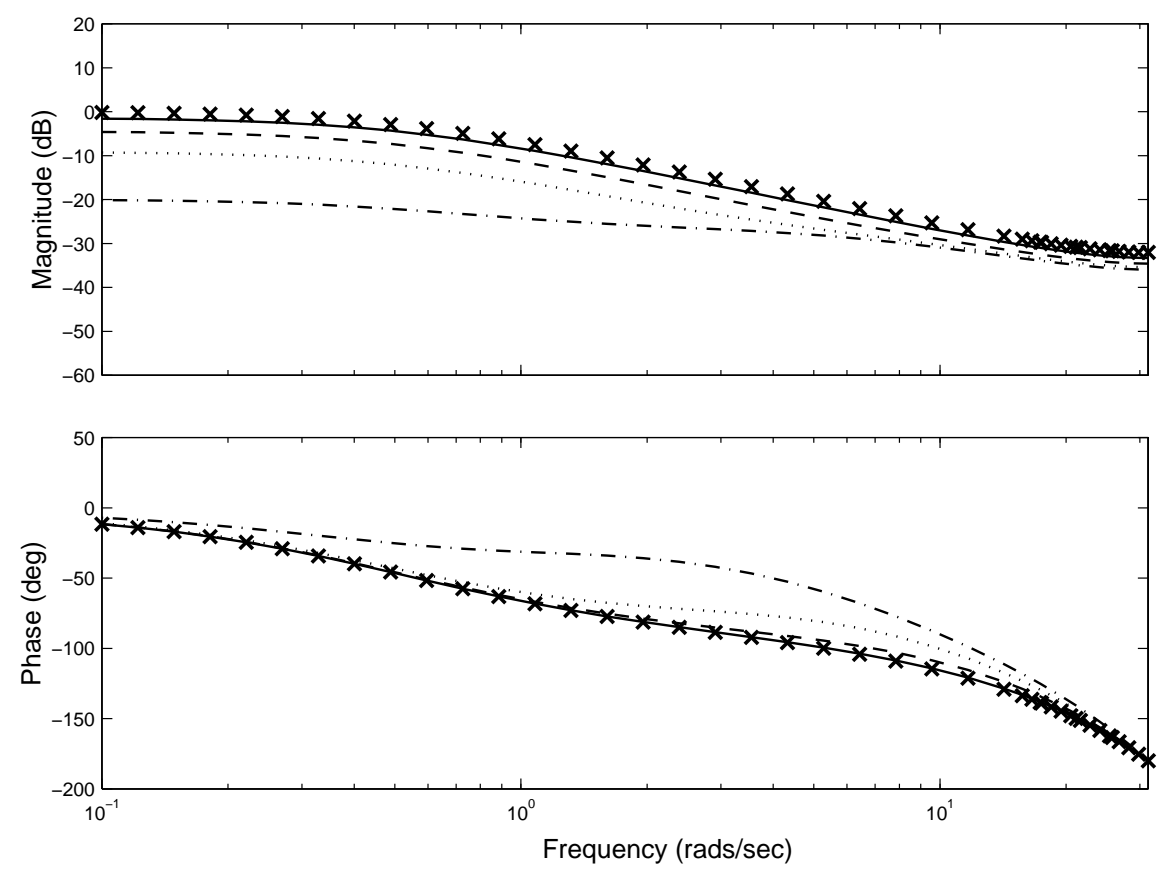

Fig. 7. Bode Plot of the True Process (x), Calculated Stationary Point for alpha $=0.15$ (solid), 0.4 (dash), 0.65 (dot) and 0.9(dash-dot).
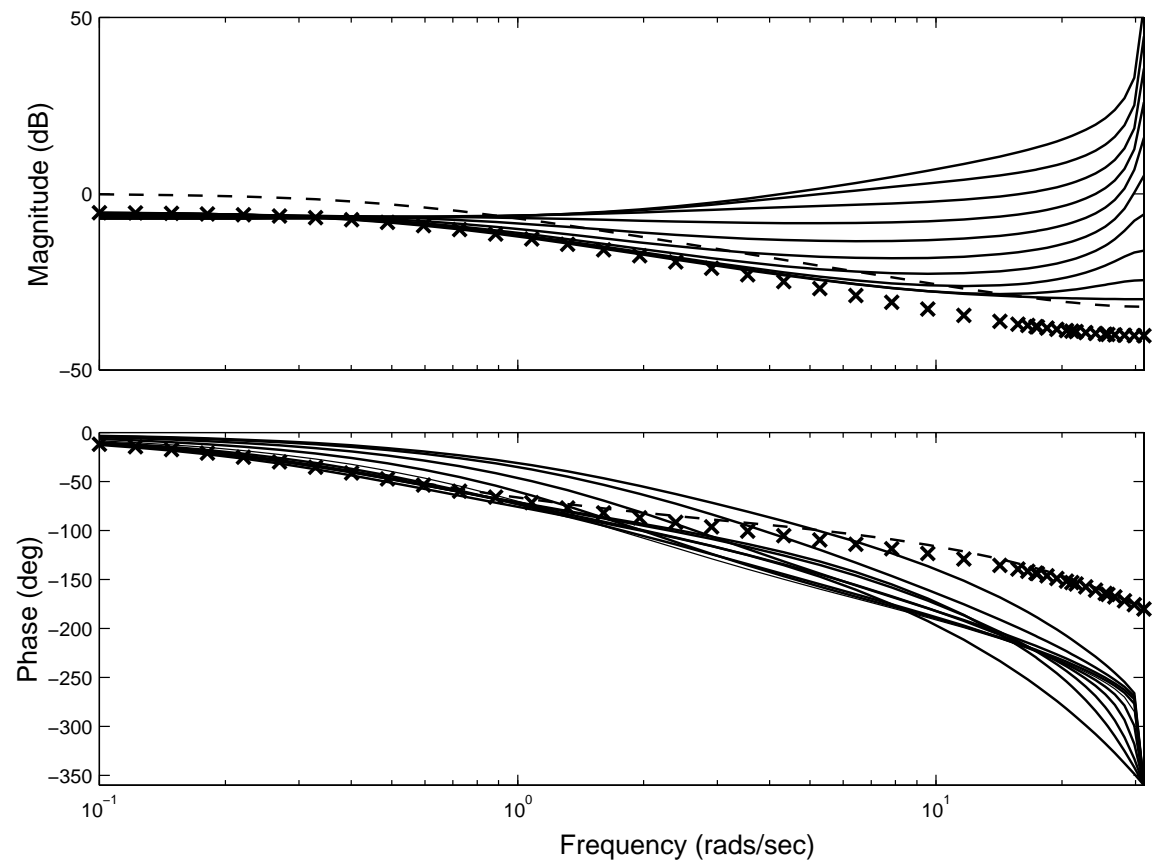

FIG. 8. Bode Plot showing divergence of the algorithm 
shows the magnitude of the discrete time closed loop poles as they evolve with respect to the iterations. Notice that during the course of the iterations, the actual closed loop system passes through unstable regions.
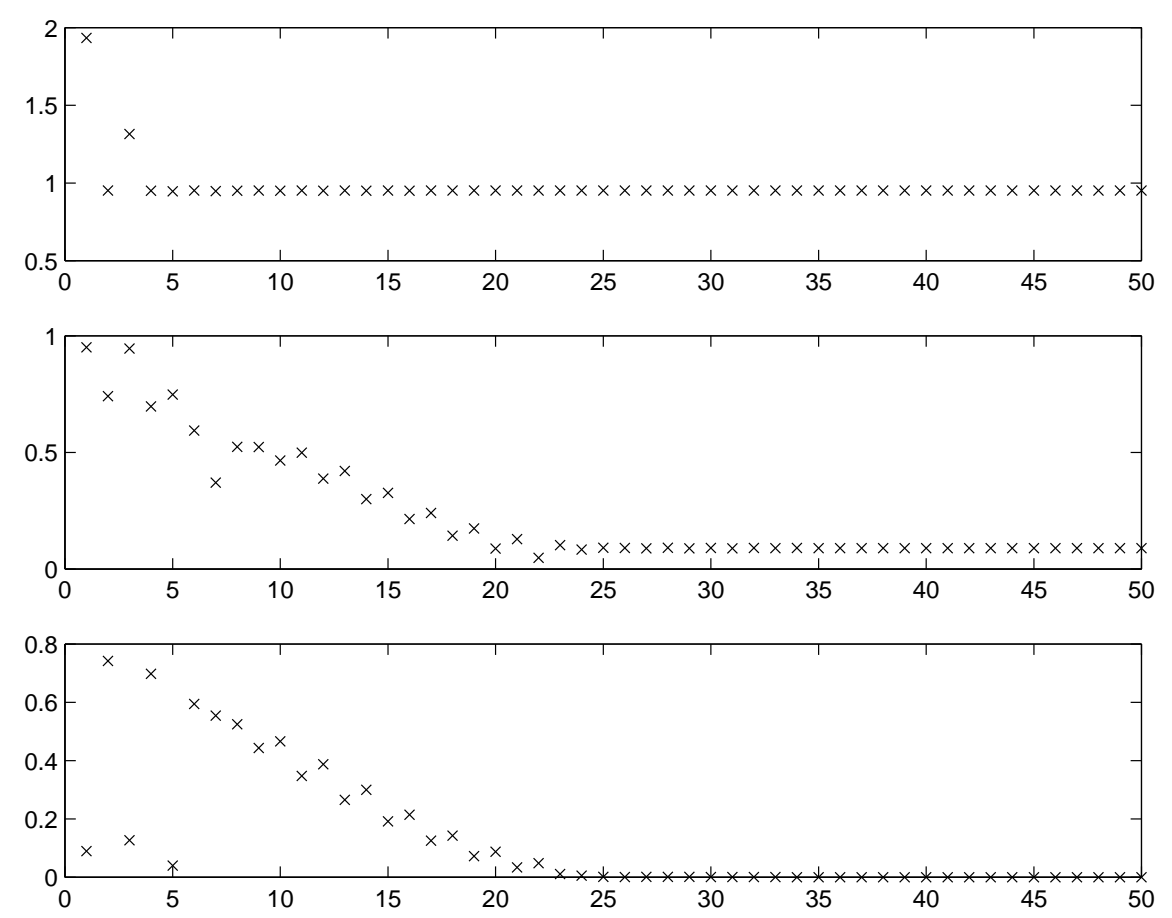

FIG. 9. Magnitude of the achieved closed loop poles Vs iterations (alpha $=0.6)$

5. Conclusion. We have considered parametric identification when operating in closed loop. Expressions have been derived for the bias when relying on self excitation (i.e. no external excitation signal applied). It is shown, that under these conditions, perfect knowledge of the noise model is required to obtain an accurate parametric estimate of the process.

This estimate has very high sensitivity to the noise model and to the properties of the driving noise itself. Indeed, the estimates are biased towards the negative inverse of the controller with noise undermodelling.

The stationary points of a simple adaptive control algorithm which relies only on self excitation were also examined. In the ideal case, where a consummate noise model exists, the estimate of the process will be perfect and hence an optimal control law can be found. However, arguably in more realistic cases it is not possible to model the noise exactly. Under these conditions the situation is more interesting.

We have shown that relatively small undermodelling in the noise model can be associated with divergence of the adaptive control algorithm even though a stationary point may exist for this algorithm. 


\section{REFERENCES}

[Astrom, 1983] K. J. Astrom, Theory and applications of adaptive control - a survey, Automatica, 19(1983), pp. 471-486.

[Astrom and Wittenmark, 1973] K. J. Astrom And B. Wittenmark, On self tuning regulators, Automatica, 9(1973), pp. 185-199.

[Astrom and Hagglund, 1995] K. J. Astrom and T. Hagglund, PID Controllers: Theory, Design and Tuning. 2nd Edition, Instrument Society of America, America, 1995.

[Churchill and Brown, 1990] R. V. Churchill And J. W. Brown, Complex Variables and Applications, McGraw-Hill, Singapore, 1990.

[Goodwin, Graebe, and Salgado, 2000] G. C. Goodwin, S. F. Graebe, and M. E. Salgado, Control System Design, Prentice Hall, Upper Saddle River, New Jersey, 2000.

[Goodwin and Sin, 1984] G. C. Goodwin And K. S. Sin, Adaptive Filtering Prediction and Control, Prentice Hall, Englewood Cliffs, New Jersey, 1984.

[Goodwin and Welsh, 2002] G. C. Goodwin And J. S. Welsh, Self excited closed loop parametric estimation in the presence of noise undermodelling, Proc. of the American Automatic Control Conference, Anchorage, Alaska, May 2002.

[Heath, 2001] W. P. HEATH, Bias of indirect non-parametric transfer function estimates for plants in closed loop, Automatica, 37:10(2001), pp. 1529-1540.

[Hjalmarsson, Gevers, and Bruyne, 1996] H. Hualmarsson, M. Gevers, and F. De Bruyne, For model-based control design, closed loop identification gives better performance, Automatica, 32:12(1996), pp. 1659-1673.

[Hjalmarsson, Gevers, and Lequin, 1998] H. Hualmarsson, M. Gevers, and O. Lequin, Iterative feedback tuning: Theory and applications, IEEE Control Systems Magazine, 18:4(1998), pp. $26-41$.

[Lee, Anderson, Kosut, and Mareels, 1993] W. S. Lee, B. D. O. Anderson, R. L. Kosut, And I. M. Y MAREels, A new approach to adaptive robust control, Int. J. of Adaptive Control and Signal Processing, 7:5(1993), pp. 183-211.

[Ljung, 1999] L. LuUng, System Identification: Theory for the User, Prentice Hall, Upper Saddle River, New Jersey, 2nd edition, 1999.

[Ljung and Forssell, 1998] L. LJUng AND U. Forssell, Bias, variance and optimal experiment design: Some comments on closed loop identification, In D. Normand-Cyrot, editor, Perspectives in Control, pp. 205-216, Springer-Verlag, 1998.

[Morari and Zafiriou, 1989] M. Morari And E. Zafiriou, Robust Process Control, Prentice Hall, Englewood Cliffs, New Jersey, 1989.

[Rhors, Valvani, Athans, and Stein, 1982] C. E. Rhors, L. Valvani, M. Athans, And G. Stein, Robustness of adaptive control algorithms in the presence of unmodeled dynamics, Proc. of the 21st IEEE Conference on Decision and Control, Florida, pp. 543-557, 1982.

[Rhors, Valvani, Athans, and Stein, 1985] C. E. Rhors, L. Valvani, M. Athans, and G. Stein, Robustness of continuous time adaptive control algorithms in the presence of unmodeled dynamics, IEEE Trans. on Automatic Control, AC-30:9(1985), pp. 881-889.

[Sastry and Bodson, 1989] S. Sastry And M. Bodson, Adaptive Control : Stability, Convergence and Robustness, Prentice Hall, Englewood Cliffs, New Jersey, 1989.

[Söderström and Stoica, 1989] T. SöDerström AND P. StoicA, System Identification, Prentice Hall, Hertfordshire (UK), 1989.

[Van den Hof and Schrama, 1995] P. M. J. VAN DEN Hof AND R. J. P. Schrama, Identification and control - closed loop issues, Automatica, 31(1995), pp. 1751-1770.

[Wellstead, 1977] P. E. Wellstead, Reference signals for closed loop identification, International Journal of Control, 26:6(1977), pp. 945-962, 1977.

[Wellstead, 1981] P. E. Wellstead, Non-parametric methods of system identification, Automatica, 
17:1(1981), pp. 55-69.

[Welsh and Goodwin, 2002] J. S. Welsh AND G. C. Goodwin, Finite sample properties of indirect non-parametric closed loop identification, IEEE Transactions on Automatic Control, 47:8(2002), pp. 1277-1292.

[Welsh and Goodwin, 2001] J. S. Welsh And G. C. Goodwin, Analysis of a self tuning iteration in the presence of noise undermodelling, Proc. of the 40th Conference on Decision and Control, pp. 739-744, December 2001.

[Zang, Bitmead, and Gevers, 1991] Z. Zang, R. R. Bitmead, And M. Gevers, $h_{2}$ iterative model refinement and control robustness enhancement, CDC 1991, Brighton, England, pp. 279284, 1991. 